\title{
Phantom Cell Analysis of Dynamic Channel Assignment in Cellular Mobile Systems
}

\author{
Kwan Lawrence Yeung and Tak-Shing Peter Yum
}

\begin{abstract}
In this paper, we propose the phantom cell analysis for dynamic channel assignment. This is an approximate analysis that can handle realistic planar systems with the threecell channel-reuse pattern. To find the blocking probability of a particular cell, two phantom cells are used to represent its six neighboring cells. Then, by conditioning on the relative positions of the two phantom cells, the blocking probability of that particular cell can be found. We found that the phantom cell analysis is not only very accurate in predicting the blocking performance, but also very computationally efficient. Besides, it is applicable to any traffic and channel-reuse patterns.
\end{abstract}

Index Terms - BDCL strategy, dynamic channel assignment, phantom cell analysis.

\section{INTRODUCTION}

$\mathbf{T}$ HERE are many studies on dynamic channel assignment (DCA) strategies and they are mainly based on computer simulations [1]-[6]. This is because in DCA, the specific set of available channels in a cell may change from time to time due to the sharing of channel resources between cells. The very complex interaction between cells makes an exact analytical solution a formidable task. An exception is an idealized strategy called "maximum packing" (MP) [7]. The MP strategy is not practical to implement since it requires system-wide information. However, MP is analytically tractable and it provides a lower bound on the overall blocking probability for all other DCA's. The modeling of MP in any realistic size network would result in a state space too large for direct computation and again computer simulation is used for performance evaluation.

Contrary to the MP, the borrowing with directional locking (BDCL) strategy [4] only needs local cells information, and it gives the lowest blocking probability among all strategies proposed in the literature that do not require system-wide information. Its four distinct features are: 1) channel ordering; 2) immediate channel reallocation; 3) directional channel locking; and 4) analytically tractable. Direct modeling of BDCL, however, would require a state space equal to $k$ times the total number of cells in the system, where $k$ is the cluster size of channel reuse. In [8], a simple, but very accurate analytical model called cell group decoupling (CGD) analysis for evaluating the performance of the BDCL strategy in linear

Manuscript received July 27, 1995; revised December 10, 1995.

K. L. Yeung is with the Department of Electronic Engineering, City University of Hong Kong, Hong Kong (e-mail: kyeung@ee.cityu.edu.hk).

T.-S. P. Yum is with the Department of Information Engineering, Chinese University of Hong Kong, Shatin, Hong Kong (e-mail: tsyum@ie.cuhk.edu.hk).

Publisher Item Identifier S 0018-9545(98)00687-2. microcellular systems was proposed. It was proved that the effect of cell group decoupling causes the blocking probability so obtained to be an upper bound and the bound was found to be very tight when compared with simulation results.

In this paper, we propose an approximate analysis for the BDCL strategy on planar microcellular systems with a threecell channel-reuse pattern. We call this phantom cell analysis. To find the blocking probability of a particular cell, two phantom cells are used to represent its six neighboring cells. Then, by conditioning on the relative positions of the two phantom cells, the blocking probability of that particular cell can be found. We formulate the problem in the next section. The call arrival rates at the phantom cells are derived in Section III. In Section IV, the approximate analytical model is established, and the product-form solution of the steady-state probabilities is derived. The analytical results are compared with simulation results in Section V and found to be very accurate in predicting the blocking performance. Besides, this analysis is applicable to any traffic and channel-reuse patterns.

\section{Problem Formulation}

Let us assume a three-cell channel-reuse pattern as an illustration of the analysis. The analysis for the seven-cell channel-reuse pattern is similar, but more complicated. Conventional cellular systems can use the three-cell reuse pattern [9] by adjusting the transmitting power of the antennas as well as their locations. Assume there are $3 m$ channels in the system divided into three nominal channel sets $A, B$, and $C$ with $m$ channels in each set. Consider a typical cell, say cell 0 in Fig. 1. Let cell 0 be allocated with nominal channel set $A$ and let its six neighbors be labeled such that cells 1, 2, and 3 are cochannel cells allocated with channel set $B$, and cells 4,5 , and 6 are cochannel cells allocated with channel set $C$. We call cells 1, 2, and 3, Group B cells, and cells 4, 5, and 6, Group $C$ cells. Let $S_{i}$ denote the set of active channels, or channels in use, in cell $i$ and $\left|S_{i}\right|$ denote the number of elements in $S_{i}$. Using the BDCL strategy [4], a call attempt at cell 0 will be blocked if and only if $\left|\sum_{k=0}^{6} S_{k}\right|=3 \mathrm{~m}$ because otherwise there will either be an idle channel in cell 0 or an idle channel can be borrowed from the neighboring cells to carry the call.

If cell 0 wants to borrow a nominal channel, say $x$, from Group $B$ cells, channel $x$ must be idle in Group $B$. Without loss of generality, assume cell 2 is currently the cell with the highest channel occupancy in Group $B$, or $\left|S_{2}\right|=$ $\max \left[\left|S_{1}\right|,\left|S_{2}\right|,\left|S_{3}\right|\right]$. Due to channel ordering and immediate channel reallocation properties of the BDCL strategy, if $x \notin$ 


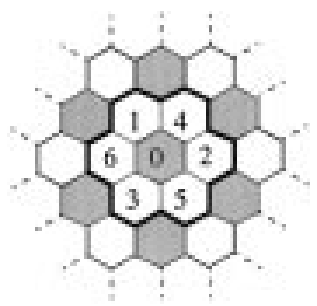

Fig. 1. A cellular system with cluster size of three.

$S_{2}$ this borrowing is granted; otherwise, the borrowing is rejected.

From the above example, we can see that whether a specific channel can be borrowed by cell 0 or not depends only on its two neighboring cells with the highest channel occupancies at that moment, one in Group $B$ and the other in Group $C$. Since these cells can be different cells in Groups $B$ and $C$ at different times, we call them phantom cells. For convenience, we denote the corresponding phantom cells of Groups $A, B$, and $C$ as cells $A, B$, and $C$. Therefore, the Markov chain model for determining the blocking probability of cell $i$ using nominal channel set $A$ for instance (i.e., cell 0 here) consists of three cells: cell $i$, phantom cell $B$, and phantom cell $C$. Similarly, for cell $j$ using channel set $B$, the Markov chain model consists of cell $j$, phantom cell $A$, and phantom cell $C$.

\section{Arrival Rates in Phantom Cellls}

Let the arrival of calls to cell $i$ be a Poisson process with rate $\lambda_{i}$ and the call duration be exponentially distributed with mean $1 / \mu$. Let random variable $M_{i}$ denote the channel occupancy of cell $i$ and $M_{A}, M_{B}$, and $M_{C}$ denote the occupancies of the phantom cells $A, B$, and $C$, respectively. Consider phantom cell $B$. Since cell $B$ is the cell with the highest channel occupancy in Group $B$ cells, its channel occupancy is given by $M_{B}=\max \left[M_{1}, M_{2}, M_{3}\right]$. Assuming the channel occupancy distributions of cells 1,2 , and 3 are independent, we have

$$
\begin{aligned}
P\left[M_{B} \leq k\right] & =P\left[M_{1} \leq k, M_{2} \leq k, M_{3} \leq k\right] \\
& =\prod_{i=1}^{3} P\left[M_{i} \leq k\right], \quad 0 \leq k \leq 3 m
\end{aligned}
$$

and thus

$$
\begin{gathered}
P\left[M_{B}=k\right]=\prod_{i=1}^{3} P\left[M_{i} \leq k\right]-\prod_{i=1}^{3} P\left[M_{i} \leq k-1\right], \\
0 \leq k \leq 3 m .
\end{gathered}
$$

The expected number of busy channels in phantom cell $B$ can be found

$$
E\left[M_{B}\right]=\sum_{k=0}^{3 m} k P\left[M_{B}=k\right]
$$

$E\left[M_{B}\right]$ can also be interpreted as the carried load of phantom cell $B$. Let $\gamma_{B}$ be the call arrival rate to phantom cell $B$.
Then in equilibrium

$$
\mu E\left[M_{B}\right]=\gamma_{B}\left(1-\mathbf{B}_{B}\right)
$$

where $\mathbf{B}_{B}$ is the blocking probability at phantom cell $B$ and is given by (12). Rearranging, we have

$$
\gamma_{B}=\frac{\mu \sum_{k=0}^{3 m} k P\left[M_{B}=k\right]}{1-\mathbf{B}_{B}} .
$$

$\gamma_{C}$ is given by a similar expression. Note that the computation of $\gamma_{B}$ and $\gamma_{C}$ requires the set of channel occupancy distributions $\left\{P\left[M_{i}=k\right]\right\}$ which is not available. In the next section, we will derive $\left\{P\left[M_{i}=k\right]\right\}$ as a function of $\gamma_{B}$ and $\gamma_{C}$. With that an iterative procedure for the simultaneous solution of $\gamma_{B}, \gamma_{C}$, and $\left\{P\left[M_{i}=k\right]\right\}$ can be started from (3) and (11) with the initial distributions $P^{(0)}\left[M_{i}=k\right]$ given by that of an $M / M / 3 m / 3 m$ queue

$$
P^{(0)}\left[M_{i}=k\right]=\left[\sum_{j=0}^{3 m} \frac{\left(\rho_{i}\right)^{j}}{j !}\right]^{-1} \frac{\left(\rho_{i}\right)^{k}}{k !}, \quad i=1,2, \cdots
$$

where $\rho_{i}=\lambda_{i} / \mu$.

\section{Blocking Probability and ChanNEl OCCUPANCY Distribution}

Consider cell 0 in Fig. 1 again. Although the two phantom cells can take different positions in Groups $B$ and $C$ at different times, they have only two relative positions: sideby-side and opposite positions. If the two phantom cells are side-by-side, a call attempt at cell 0 will be blocked if and only if $\left|S_{0}\right|+\left|S_{B}\right|+\left|S_{C}\right|=3 \mathrm{~m}$. In other words, the total number of active calls in cells $0, B$, and $C$ cannot be greater than $3 \mathrm{~m}$. On the other hand, if the two phantom cells are opposite to each other, a call attempt at cell 0 will be blocked if and only if $\left|S_{0} \cup S_{B} \cup S_{C}\right|=3 \mathrm{~m}$. In this case, the total number of active channels in cells $0, B$, and $C$ can exceed $3 m$ and in the extreme case, a total of $6 \mathrm{~m}$ active channels can be supported when $\left|S_{0}\right|=0$.

Let $\alpha$ and $1-\alpha$ be the probability that the two phantom cells $B$ and $C$ are at opposite and side-by-side positions, respectively. Then, the blocking probability $\mathbf{B}_{0}$ at cell 0 can be expressed as

$$
\mathbf{B}_{0}=\alpha B_{\text {opp }}+(1-\alpha) B_{\text {side }}
$$

where $B_{\text {side }}$ and $B_{\text {opp }}$ are the respective conditional blocking probabilities.

\section{A. Derivation of $\alpha$}

Let $f_{i}$ be the probability that cell $i$ is a phantom cell. Consider the Group $B$ cells, i.e., cells 1, 2, and 3 in Fig. 1. 


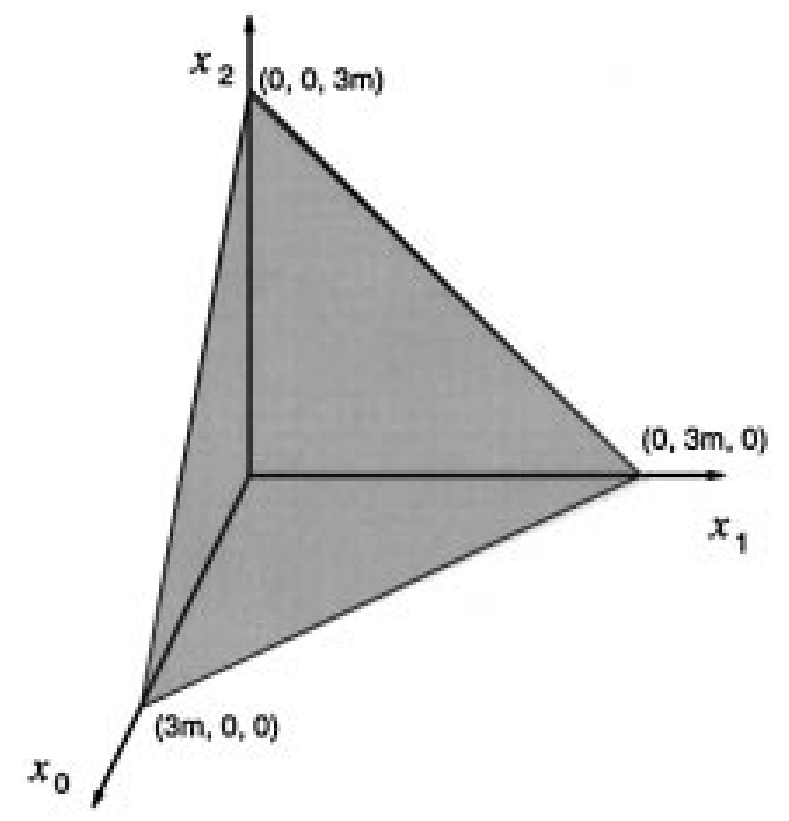

Fig. 2. State space of the Markov chain for phantom cells at side-by-side positions.

Then $f_{1}$ the probability that cell 1 is the phantom cell of Group $B$ cells (or cell $B$ ) can be derived from the definition of phantom cells to be

$$
\begin{aligned}
f_{1}= & \sum_{k=0}^{3 m}\left\{P\left[M_{2}<k\right] P\left[M_{3}<k\right]\right. \\
& +\frac{1}{2} P\left[M_{2}=k\right] P\left[M_{3}<k\right] \\
& +\frac{1}{2} P\left[M_{2}<k\right] P\left[M_{3}=k\right] \\
& \left.+\frac{1}{3} P\left[M_{2}=k\right] P\left[M_{3}=k\right]\right\} P\left[M_{1}=k\right] .
\end{aligned}
$$

Note that in (5) we have assumed that when two or more cells have the same highest channel occupancy, the phantom cell is picked with equal probabilities. This accounts for the last three terms on the right hand side of (5). $f_{i}$ (for $i=2,3, \cdots, 6$ ) can be similarly found. From the actual cellular layout in Fig. 1, it is seen that cell 1 is opposite to cell 5 , cell 2 to cell 6 , and cell 3 to cell 4 . Therefore, the probability that the phantom cells $B$ and $C$ are at opposite positions is given by

$$
\alpha=f_{1} f_{5}+f_{2} f_{6}+f_{3} f_{4} .
$$

\section{B. Derivation of $B_{\text {side }}$}

Let $p\left(x_{0}, x_{1}, x_{2}\right)$ be the steady-state probability that $\left|S_{0}\right|=$ $x_{0},\left|S_{B}\right|=x_{1}$, and $\left|S_{C}\right|=x_{2}$ given that the two phantom cells are at side-by-side positions. To calculate $B_{\text {side, the }}$ blocking probability of cell 0 when the two phantom cells $B$ and $C$ are at side-by-side positions, we need to solve the three-dimensional (3-D) Markov chain shown in Fig. 2. This Markov chain belongs to the coordinate convex type for which product-form solution can be obtained [10]. Let $a_{0}=\lambda_{0} / \mu$, $a_{1}=\lambda_{B} / \mu$, and $a_{2}=\lambda_{C} / \mu$. As seen from Fig. 2 , the total number of states $N$ can be obtained by adding the number of states on each of the $\left(x_{1}, x_{2}\right)$ planes, one for each value of

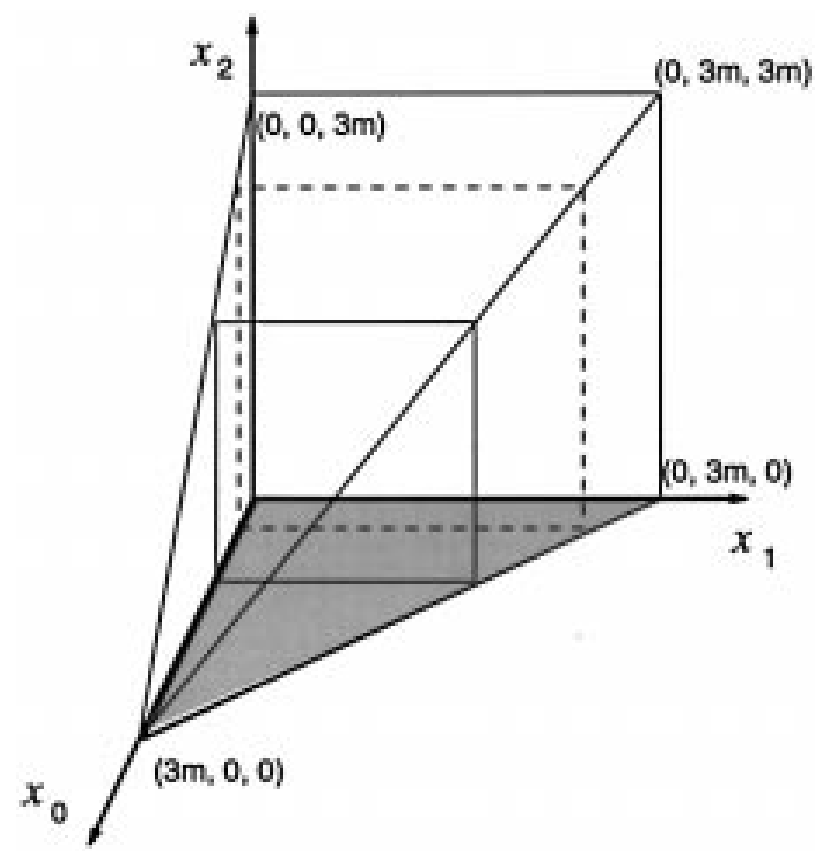

Fig. 3. State space of the Markov chain for phantom cells at opposite positions.

$x_{0}$. In other words

$$
\begin{aligned}
N= & 1+[1+2]+[1+2+3]+\cdots+[1+2+\cdots+3 m] \\
& +[1+2+\cdots+(3 m+1)] \\
= & 1+3+6+10+\cdots+\frac{(3 m+1)(3 m+2)}{2} \\
= & \frac{1}{2}\left(9 m^{3}+18 m^{2}+11 m+2\right) .
\end{aligned}
$$

The steady-state probability $p\left(x_{0}, x_{1}, x_{2}\right)$ is given by

$$
p\left(x_{0}, x_{1}, x_{2}\right)=G^{-1} \prod_{j=0}^{2} \frac{a_{j}^{x_{j}}}{x_{j} !}
$$

where

$$
G=\sum_{x_{0}+x_{1}+x_{2} \leq 3 m}\left(\prod_{j=0}^{2} \frac{a_{j}^{x_{j}}}{x_{j} !}\right) .
$$

The blocking probability $B_{\text {side }}$ is obtained as

$$
B_{\text {side }}=\sum_{x_{0}+x_{1}+x_{2}=3 m} p\left(x_{0}, x_{1}, x_{2}\right) \text {. }
$$

\section{Derivation of $B_{\mathrm{opp}}$}

If the phantom cells $B$ and $C$ are at opposite positions, the channel usage in cells $0, B$, and $C$ can be described by the 3-D Markov chain shown in Fig. 3. For $x_{0}=i(0 \leq i \leq 3 m+1)$, the two-dimensional (2-D) state space of $\left(x_{1}, x_{2}\right)$ has a square shape with a total of $(3 m-i+1)^{2}$ states. The total number of states $N^{\prime}$ in the square prism of Fig. 3 is

$$
N^{\prime}=\sum_{i=0}^{3 m+1}(3 m-i+1)^{2}=\frac{1}{2}\left(18 m^{3}+27 m^{2}+13 m+2\right) \text {. }
$$




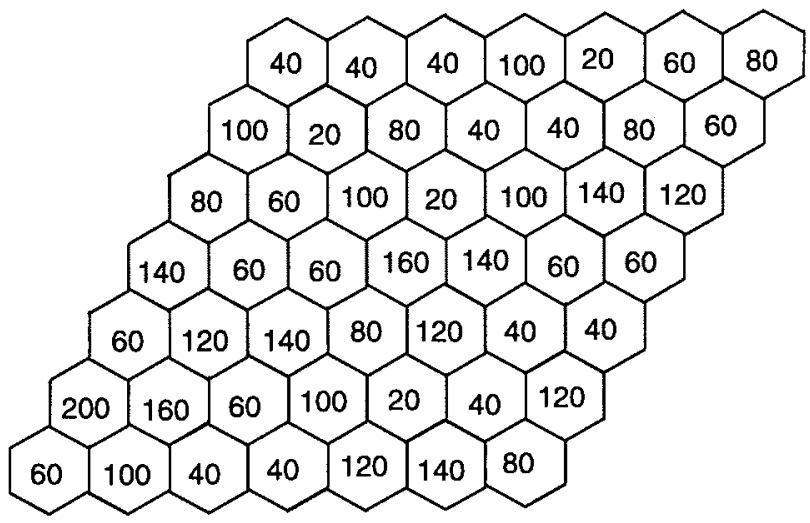

(a)

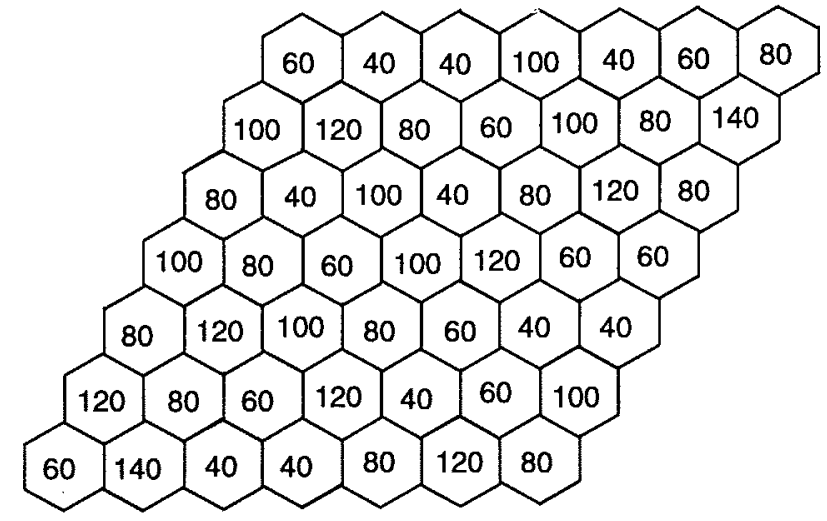

(b)

Fig. 4. Cellular system with nonuniform traffic distributions.

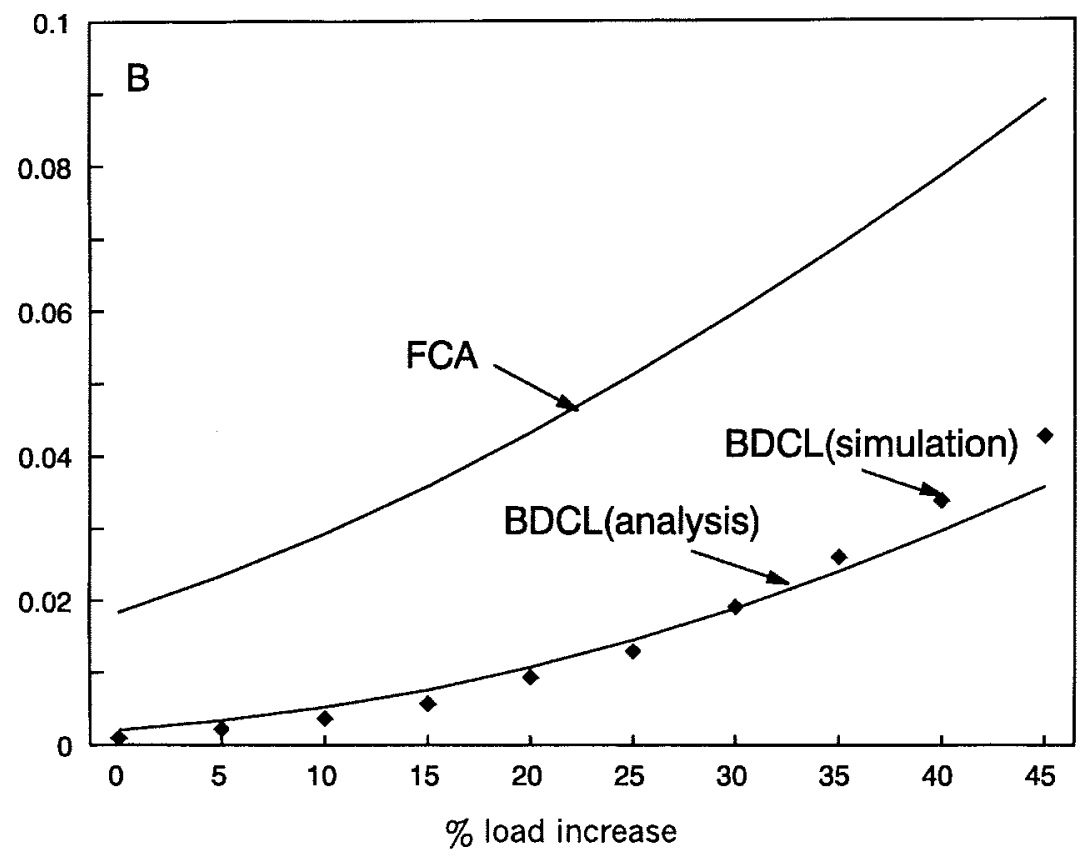

Fig. 5. Uniform traffic distribution with 100 calls/h/cell.

The product-form solution of the steady-state probability $q\left(x_{0}, x_{1}, x_{2}\right)$ is given by

$$
q\left(x_{0}, x_{1}, x_{2}\right)=G^{-1} \prod_{j=0}^{2} \frac{a_{j}^{x_{j}}}{x_{j} !}
$$

where

$$
G=\sum_{x_{0}+x_{1} \leq 3 m, x_{0}+x_{2} \leq 3 m}\left(\prod_{j=0}^{2} \frac{a_{j}^{x_{j}}}{x_{j} !}\right) .
$$

The blocking probability $B_{\text {opp }}$ is obtained as

$$
\begin{aligned}
B_{\mathrm{opp}}= & \sum_{x_{0}+x_{2} \leq 3 m} q\left(x_{0}, 3 m-x_{0}, x_{2}\right) \\
& +\sum_{x_{0}+x_{1}<3 m} q\left(x_{0}, x_{1}, 3 m-x_{0}\right) .
\end{aligned}
$$

Substituting (3), (7), and (9) into (4), the blocking probability at cell 0 is found. The overall blocking probability of a system consisted of $N$ cells is simply

$$
\mathbf{B}=\left(\sum_{k=0}^{N-1} \lambda_{k}\right)^{-1} \sum_{k=0}^{N-1} \lambda_{k} \mathbf{B}_{k}
$$

where $\mathbf{B}_{k}$, the call blocking probability at cell $k$, is given by the same expression in (4).

\section{Channel Occupancy Distribution}

Let $P\left[M_{i}=k \mid\right.$ side $]$ and $P\left[M_{i}=k \mid\right.$ opp $]$ be the probabilities that cell $i$ has occupancy $k$ given that the two phantom cells are at side-by-side and opposite positions, respectively. From (6) and (8), we have

$$
\begin{aligned}
& P\left[M_{i}=k \mid \text { side }\right]=\sum_{x_{1}, x_{2}} p\left(k, x_{1}, x_{2}\right) \\
& P\left[M_{i}=k \mid \mathrm{opp}\right]=\sum_{x_{1}, x_{2}} q\left(k, x_{1}, x_{2}\right) .
\end{aligned}
$$




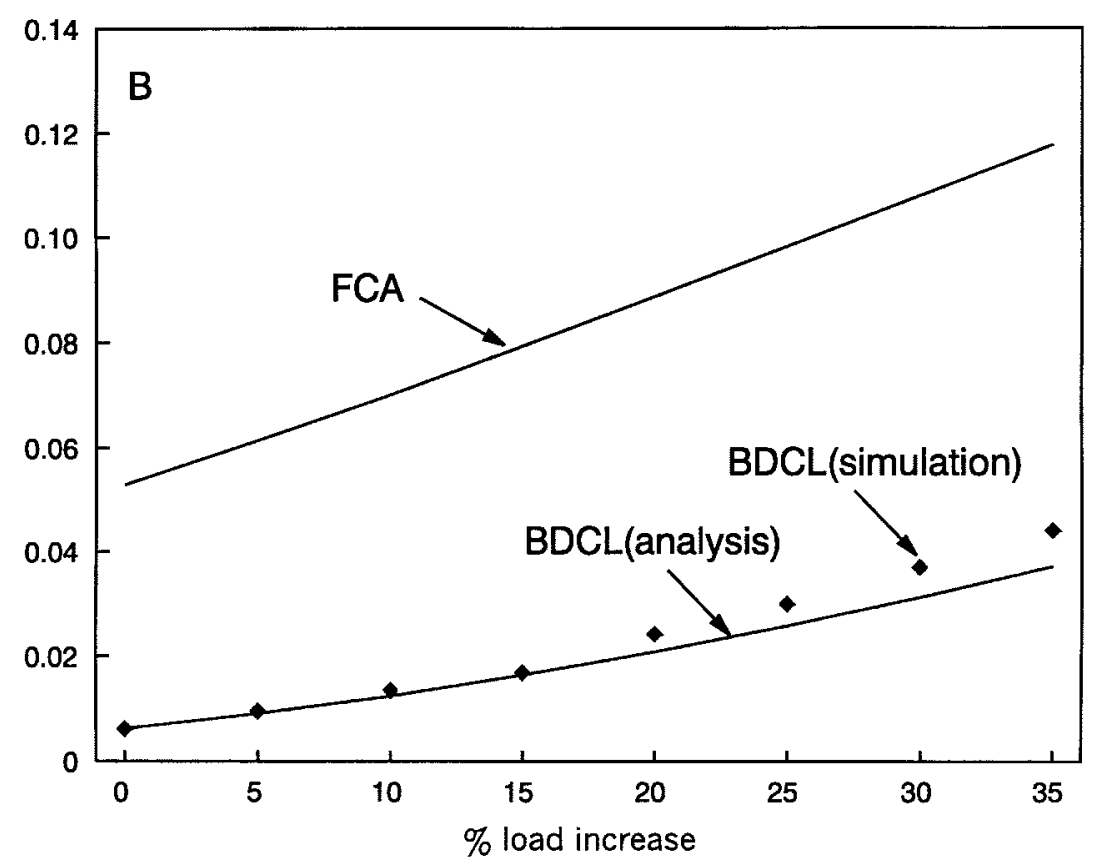

Fig. 6. Nonuniform traffic distribution A.

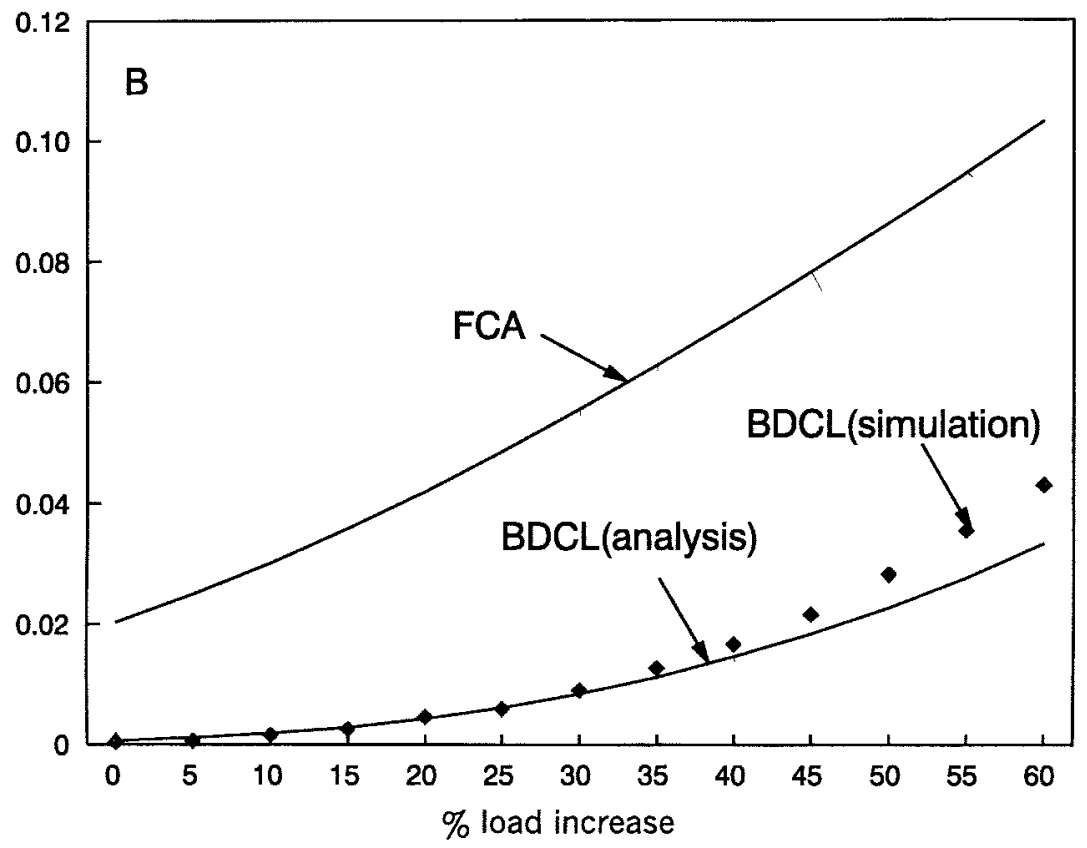

Fig. 7. Nonuniform traffic distribution B.

By the law of total probability, we have

$$
P\left[M_{i}=k\right]=\alpha P\left[M_{i}=k \mid \mathrm{opp}\right]+(1-\alpha) P\left[M_{i}=k \mid \text { side }\right] .
$$

The blocking probability of phantom cell $B$ can be found as follows. Let $P B\left[M_{i}=k\right]$ be the blocking probability of cell $i$ with channel occupancy $k$. Let $P B\left[M_{i}=k \mid\right.$ side $]$ and $P B\left[M_{i}=k \mid\right.$ opp $]$ be the blocking probabilities of cell $i$ with channel occupancy $k$ given that the two phantom cells are at side-by-side and opposite positions respectively. From (6) and
(8) again, we have

$$
\begin{aligned}
P B\left[M_{i}=k \mid \text { side }\right]= & \sum_{x_{1}+x_{2}=3 m-k} p\left(k, x_{1}, x_{2}\right) \\
P B\left[M_{i}=k \mid \mathrm{opp}\right]= & \sum_{x_{2} \leq 3 m-k} q\left(k, 3 m-k, x_{2}\right) \\
& +\sum_{x_{1}<3 m-k} q\left(k, x_{1}, 3 m-k\right) .
\end{aligned}
$$

Then

$$
\begin{aligned}
P B\left[M_{i}=k\right]= & \alpha P B\left[M_{i}=k \mid \mathrm{opp}\right] \\
& +(1-\alpha) P B\left[M_{i}=k \mid \text { side }\right] .
\end{aligned}
$$


$\mathbf{B}_{B}$, the blocking probability at phantom cell $B$, is given by

$$
\begin{aligned}
\mathbf{B}_{B} & =\sum_{k=0}^{3 m} P B\left[M_{B}=k\right] P\left[M_{B}=k\right] \\
& =\sum_{k=0}^{3 m} \sum_{i=1}^{3} f_{i} P B\left[M_{i}=k\right] P\left[M_{B}=k\right]
\end{aligned}
$$

where $f_{i}$ is given in (5).

\section{NUMERICAL RESULTS}

In this section, we investigate the performance of the phantom cell analysis by considering a cellular system consisting of 49 cells as shown in Fig. 4. Let the number of nominal channels $m$ in each cell be ten. Let the arrival of calls be a Poisson process and the call duration be exponentially distributed with mean $3 \mathrm{~min}$. Each simulation data point is obtained by collecting statistics of 510000 calls with the initial 10000 calls discarded.

First, we examine the performance under uniform traffic distribution. The base load of each cell is first engineered at 100 calls/h. The blocking probability $\mathbf{B}$ is plotted in Fig. 5 against the increase in traffic load. The Erlang- $B$ curve corresponding to the fixed channel assignment with ten channels per cell is plotted for comparison. It is seen that the analytical result is very close to the simulation.

Next, we consider two nonuniform traffic distributions with the base traffic in calls/hour of each cell shown in Fig. 4(a) and (b). The analytical and simulation results for BDCL are plotted in Figs. 6 and 7. The curves corresponding to FCA are again obtained by Erlang- $B$ formula with ten channels per cell. For both traffic distributions, very close agreements between analytical and simulation results are found. These results show that the phantom cell analysis is accurate under very different traffic conditions.

\section{CONCLUSIONS}

An approximate analytical model called phantom cell analysis for evaluating the performance of the BDCL strategy was proposed in this paper. The phantom cell analysis was found to be very computationally efficient and accurate. It is applicable to any traffic patterns and any cellular layouts. In this paper, only systems with a three-cell channel-reuse pattern were considered. The analysis of systems with sevencell channel-reuse pattern would require the solution of many seven-dimensional (7-D) Markov chains, and this is still a formidable task with today's computational power. The effect of DCA on handoff performance is a research topic that requires further work.

\section{REFERENCES}

[1] V. H. MacDonald, "Advanced mobile phone service: The cellular concept,” Bell Syst. Tech. J., vol. 58, pp. 15-41, Jan. 1979.

[2] T. J. Kahwa and N. D. Georganas, "A hybrid channel assignment scheme in large-scale, cellular-structured mobile communication systems," IEEE Trans. Commun., vol. COM-26, no. 4, pp. 432-438, 1978.

[3] S. M. Elnoubi, R. Singh, and S. C. Gupta, "A new frequency channel assignment in high capacity mobile communication systems," IEEE Trans. Veh. Technol., vol. VT-31, no. 3, pp. 125-131, 1982.

[4] M. Zhang and T. S. Yum, "Comparisons of channel assignment strategies in cellular mobile telephone systems," IEEE Trans. Veh. Technol., vol. 38, pp. 211-215, Nov. 1989.

[5] L. G. Anderson, "A simulation study of some dynamic channel assignment algorithms in a high capacity mobile telecommunications system," IEEE Trans. Veh. Technol., vol. VT-22, pp. 210-217, Nov. 1973.

[6] D. C. Cox and D. O. Reudink, "Increasing channel occupancy in largescale mobile radio systems: Dynamic channel reassignment," IEEE Trans. Veh. Technol., vol. VT-22, pp. 218-222, Nov. 1973.

[7] D. Everitt and D. Manfield, "Performance analysis of cellular mobile communication systems with dynamic channel assignment," IEEE $J$. Select. Areas Commun., vol. 7. no. 8, pp. 1172-1180, 1989.

[8] K. L. Yeung and T. S. Yum, "Cell group decoupling analysis of a channel borrowing based dynamic channel assignment strategy in linear radio systems," IEEE Trans. Commun., vol. 43, pp. 1289-1292, Apr. 1995.

[9] W. C. Y. Lee, "Smaller cells for greater performance," IEEE Commun. Mag., vol. 30, no. 11, pp. 19-23, 1991.

[10] J. S. Kaufman, "Blocking in a shared resource environment," IEEE Trans. Commun., vol. COM-29, no. 10, pp. 1474-1481, 1981.

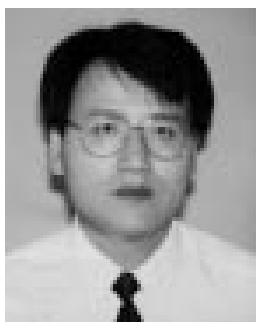

Kwan Lawrence Yeung received the B.Eng. and $\mathrm{Ph} . \mathrm{D}$. degrees in information engineering from the Chinese University of Hong Kong, Hong Kong, in 1992 and 1995, respectively.

In May 1995, he joined the City University of Hong Kong as an Assistant Professor. His research interests include personal and mobile communication systems, high-speed networking, broadband packet switches, and lightwave networks.

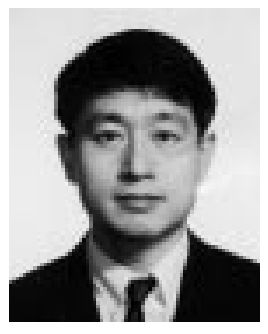

Tak-Shing Peter Yum received the B.Sc., M.Sc., M.Ph., and Ph.D. degrees from Columbia University, NY, in 1974, 1975, 1977, and 1978, respectively.

He worked in Bell Telephone Laboratories for two and one-half years and taught at the National Chiao Tung University, Taiwan, R.O.C., for two years before joining the Chinese University of Hong Kong, Hong Kong, in 1982. He has published original research on packet-switched networks with contributions in routing algorithms, buffer management, deadlock detection algorithms, message resequencing analysis, and multiaccess protocols. In recent years, he branched out to work on the design and analysis of cellular network, lightwave networks, and video-distribution networks. He believes that the next challenge is designing an intelligent network that can accommodate the needs of individual customers. 